\title{
NanoLuc Bioluminescence-Driven Photodynamic Activation of Cholecystokinin 1 Receptor with Genetically-Encoded Protein Photosensitizer MiniSOG
}

\author{
Yuan Li and Zong Jie Cui *(1) \\ Institute of Cell Biology, Beijing Normal University, Beijing 100875, China; liyuanswkx@163.com \\ * Correspondence: zjcui@bnu.edu.cn; Tel.: +86-10-5880-9162
}

Received: 26 April 2020; Accepted: 25 May 2020; Published: 26 May 2020

\begin{abstract}
In contrast to reversible activation by agonist, cholecystokinin 1 receptor (CCK1R) is permanently activated by singlet oxygen generated in photodynamic action, with sulphonated aluminium phthalocyanine or genetically encoded mini singlet oxygen generator (miniSOG) as photosensitizer. In these works, a halogen light source was used to power photodynamic action. For possible in vivo application of photodynamic CCK1R physiology, bearing a cumbersome light-delivery device connected to an external light source by experimental animals might interfere with their behavior. Therefore, in the present work, the possibility of bioluminescence-driven miniSOG photodynamic CCK1R activation was examined, as monitored by Fura-2 calcium imaging. In parallel experiments, it was found that, after plasma membrane (PM)-localized expression of miniSOGPM in AR4-2J cells, light irradiation with blue light-emitting diode (LED) $\left(450 \mathrm{~nm}, 85 \mathrm{~mW} \cdot \mathrm{cm}^{-2}, 1.5 \mathrm{~min}\right)$ induced persistent calcium oscillations that were blocked by CCK1R antagonist devazepide $2 \mathrm{nM}$. NanoLuc was expressed bicistronically with miniSOG $_{\mathrm{PM}}$ via an internal ribosome entry site (IRES) sequence (pminiSOG ${ }_{P M}$-IRES-NanoLuc). The resultant miniSOG ${ }_{P M}$-IRES-NanoLuc-AR4-2J cells were found to generate strong bioluminescence upon addition of NanoLuc substrate coelenterazine. Strikingly, coelenterazine 5 microM was found to trigger long-lasting calcium oscillations (a hallmark for permanent CCK1R activation) in perifused miniSOG ${ }_{P M}$-IRES-NanoLuc-AR4-2J cells. These data indicate that NanoLuc bioluminescence can drive miniSOGPM photodynamic CCK1R activation, laying the foundation for its future in vivo applications.
\end{abstract}

Keywords: miniSOG; CCK1 receptor; NanoLuc; ${ }^{1} \mathrm{O}_{2}$; calcium oscillations

\section{Introduction}

The rhodopsin-type or A class calcium-mobilizing G protein-coupled receptor (GPCR) cholecystokinin type 1 (CCK1 or CCK1R) [1,2] is widely expressed both in the central nervous system (CNS) [3-6] and in peripheral tissues [7-12]. CCK1R in the CNS regulates neurotransmitter release [13-15], anxiety [16,17], appetite [18], neurogenesis [19], brain development [6], learning, and memory formation [20]. Peripheral CCK1R is involved in pancreatic [21,22] and adrenocortical secretion [23], gallbladder contraction [21], inhibition of gastric emptying and gastric acid secretion [24], lower esophageal sphincter relaxation [25], and slowing of colonic motility [26].

Owing to the wide distribution and participation in important physiological functions, CCK1R and coupled signaling pathways are rather well characterized [27], including coupling to calcium signaling $[1,2,28]$. In contrast to reversible activation by agonists, one strikingly conspicuous feature of CCK1 receptor pharmacology is that CCK1R such as those present at the basal plasma membrane in rat pancreatic acini are permanently activated by the lowest lying excited state of molecular oxygen, the delta singlet oxygen $\left({ }^{1} \mathrm{O}_{2}\right)$ [29-32]. 
We have previously found that photodynamically generated ${ }^{1} \mathrm{O}_{2}$ with chemical photosensitizers sulphonated aluminum phthalocyanine (SALPC) or gadolinum porphyrin-like macrocycle B (GdPLMB) permanently activated CCK1R endogenously expressed in rat pancreatic acinar cells [29-31] or ectopically expressed in human embryonic kidney epithelial cell HEK293 [33]. Emergence in recent years of genetically-encoded protein photosensitizers (GEPPs) such as KillerRed or mini singlet oxygen generator (miniSOG) prompted us to extend our earlier works with chemical photosensitizers to these new GEPPs. We have found that the GEPPs KillerRed or miniSOG specifically expressed at the plasma membrane in rat pancreatic acinar tumor cell AR4-2J were able after light irradiation with a halogen cold light source to photodynamically activate CCK1R permanently [33].

The miniSOG is the first GEPP known to specifically generate ${ }^{1} \mathrm{O}_{2}$ (quantum yield $\Phi_{\Delta} \geq 0$.03) [34-36]. MiniSOG variants and other flavin-binding ${ }^{1} \mathrm{O}_{2}$-generating protein photosensitizers with varied ${ }^{1} \mathrm{O}_{2}$ quantum yields (up to 0.61 ) have also emerged [32,37-40].

The availability of a large arsenal of GEPPs might immediately enable in vivo study of photodynamic CCK1R physiology or pharmacology in CCK1R-expressing cells in both the CNS and peripheral tissues. However, a major challenge for such works might be the possible interference from bearing a cumbersome light-delivery device connected to an external light source, of the intrinsic animal behavior or function under investigation. Therefore, in the present work, the possibility of bioluminescence-driven miniSOG photodynamic CCK1R activation was examined. In parallel experiments, it was found that light irradiation with a blue light-emitting diode (LED) of AR4-2J cells expressing plasma membrane (PM)-localized miniSOG (miniSOG ${ }_{P M}$ ) resulted in persistent calcium oscillations that were completely blocked by CCK1R antagonist devazepide. When miniSOG $\mathrm{PM}_{\text {was }}$ expressed bicistronically with a miniaturized luciferase, NanoLuc, by way of an internal ribosome entry site (IRES, to ensure separate protein expression of NanoLuc and miniSOG) sequence, the resultant miniSOG ${ }_{P M}$-IRES-NanoLuc-AR4-2J cells emitted strong bioluminescent light upon addition of NanoLuc substrate coelenterazine. Addition of coelenterazine at micromolar concentrations to perifused miniSOG ${ }_{\mathrm{PM}}$-IRES-NanoLuc-AR4-2J cells was found to trigger persistent cytosolic calcium oscillations, which lasted long after wash-out of substrate coelenterazine. These data indicate that NanoLuc bioluminescence could drive miniSOG photodynamic CCK1R activation, laying the foundation for future in vivo applications.

\section{Results}

Fusion of a PM-localizing sequence (see Materials and Methods) to miniSOG (Figure 1a) obtained a PM-targeting miniSOG, miniSOG ${ }_{P M}$. After transduction of AR4-2J cells with plasmid pminiSOG ${ }_{P M}$, protein expression was confirmed by miniSOG ${ }_{\mathrm{PM}}$ fluorescence microscopy (Figure 1b) and the effect of light irradiation was examined. In control non-transfected AR4-2J cells, cytosolic calcium concentration remained rather stable, and blue LED light irradiation $\left(450 \mathrm{~nm}, 85 \mathrm{~mW} \cdot \mathrm{cm}^{-2}, 1.5 \mathrm{~min}\right)$ induced no major effect (Figure 1c). In control non-transfected AR4-2J cells, perifusion with chemical photosensitizer SALPC $(1 \mu \mathrm{M})$ in the dark had no effect; after wash-out of unbound SALPC, subsequent light irradiation with red light $\left(\lambda>580 \mathrm{~nm}, 36.7 \mathrm{~mW} \cdot \mathrm{cm}^{-2}, 1.5 \mathrm{~min}\right)$ from a halogen cold light source triggered calcium oscillations that persisted long after cessation of light illumination (Figure 1d). Light irradiation with blue LED ( $450 \mathrm{~nm}, 85 \mathrm{~mW} \cdot \mathrm{cm}^{-2}, 1.5 \mathrm{~min}$ ) of miniSOG ${ }_{\mathrm{PM}^{-}}$-AR4-2J cells triggered similar long-lasting or persistent calcium oscillations (Figure 1e), which were completely blocked reversibly by the CCK1R antagonist devazepide $2 \mathrm{nM}$ (Figure 1f). These data together indicate that permanent miniSOGPM photodynamic activation of CCK1R could be driven by blue LED $(450 \mathrm{~nm})$ light, to trigger persistent calcium oscillations in miniSOG ${ }_{\mathrm{PM}}$-AR4-2J cells. 
$a$

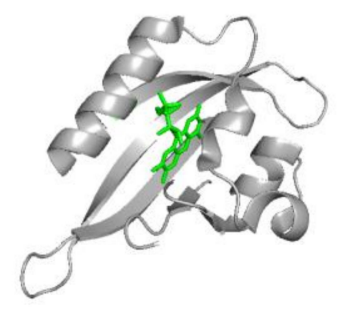

b
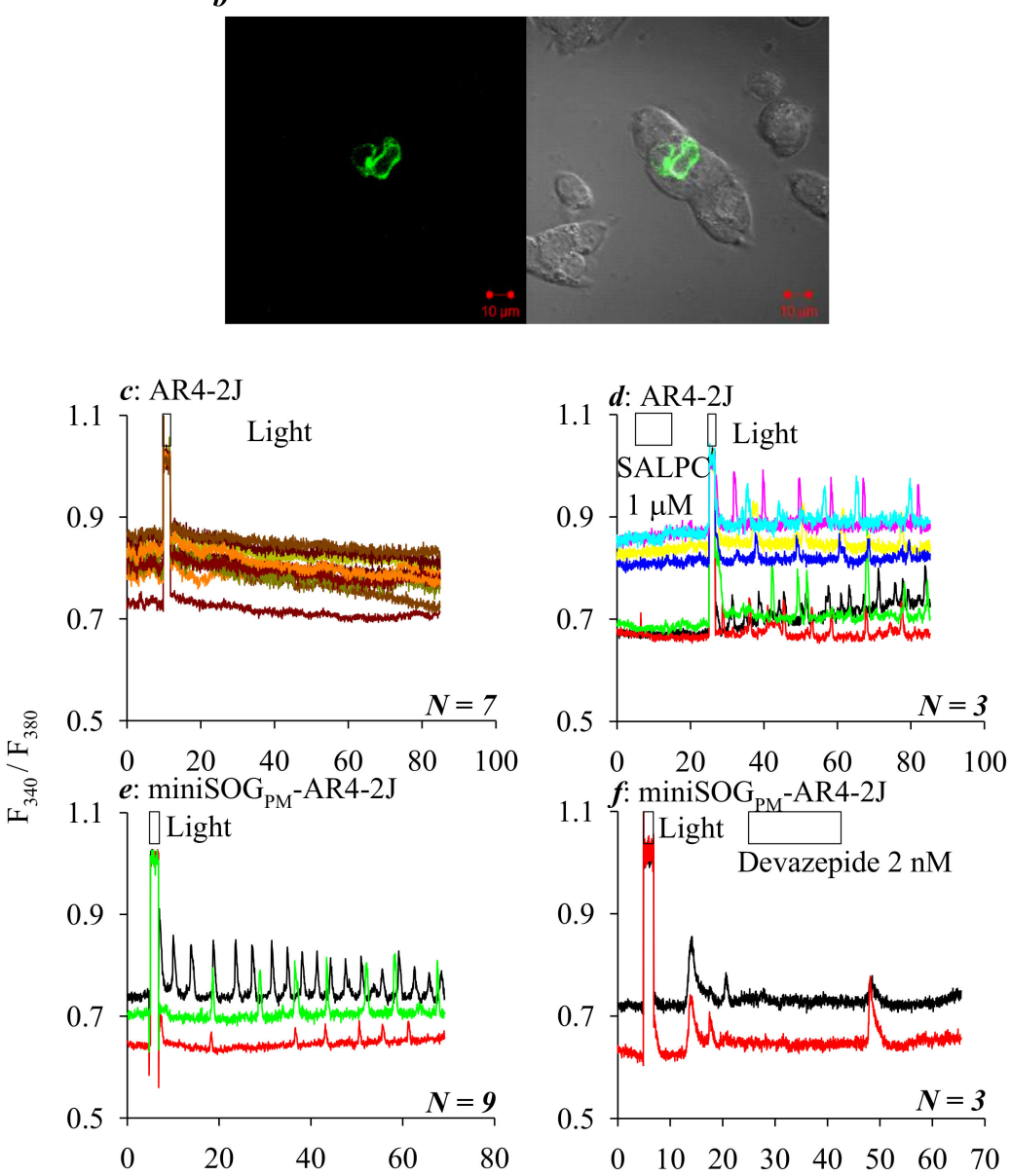

Time (min)

Figure 1. Mini singlet oxygen generator (miniSOGPM) photodynamic activation of CCK1R in AR4-2J cells driven by a blue LED light source. (a) Structure of miniSOG. The full amino acid sequence of miniSOG is from [37], and three-dimensional model built by Swiss-model. The miniSOG chromophore flavin mononucleotide (FMN) is outlined in green. (b) MiniSOGPM expression in AR4-2J, confocal images ( $\lambda$ ex $488 \mathrm{~nm}$ ) were taken $24 \mathrm{~h}$ after transfection. Control non-transfected AR4-2J cells (c, $N=7 ; \mathbf{d}, N=3$ ) or miniSOGPM-AR4-2J cells $(\mathbf{e}, N=9 ; \mathbf{f}, N=3)$ loaded with Fura-2 AM were perifused, and sulphonated aluminum phthalocyanine (SALPC) $1 \mu \mathrm{M}$, devazepide $2 \mathrm{nM}$, red light (>580 $\mathrm{nm}, 36.7 \mathrm{~mW} \cdot \mathrm{cm}-2,1.5 \mathrm{~min}$ ) from a halogen cold light source (d), or blue light-emitting diode (LED) $(450 \mathrm{~nm}, 85 \mathrm{~mW} \cdot \mathrm{cm}-2,1.5 \mathrm{~min})$ light (c,e,f) were applied as indicated. (c) Non-transfected AR4-2J cells with blue LED light irradiation. (d) Non-transfected AR4-2J cells exposed to SALPC $1 \mu \mathrm{M}$, followed by red light irradiation from halogen cold light source. (e,f) MiniSOGPM-AR4-2J cells with blue LED light irradiation. Note the complete inhibition of calcium oscillations by cholecystokinin 1 receptor (CCK1R) antagonist devazepide $2 \mathrm{nM}(\mathbf{f}, N=3)$. Colored calcium traces tracings are shown with each from individual cells measured simultaneously. These original tracings shown are from 1 out of $N$ (as indicated) identical experiments. 
In the above experiments, for miniSOG ${ }_{\mathrm{PM}}$ photodynamic CCK1R activation to occur, an external light source, blue LED $(450 \mathrm{~nm})$, was applied at a power density of $85 \mathrm{~mW} \cdot \mathrm{cm}^{-2}$ for $1.5 \mathrm{~min}$. For possible in vivo applications, it would be desirable if one could make use of an internal light source, namely, bioluminescence. Will bioluminescence generated by NanoLuc be strong enough to power miniSOGPM photodynamic CCK1R activation? It was found that, when miniSOG and NanoLuc were co-expressed in a bicistronic vector in AR4-2J cells, both proteins retained their full function. The expressed NanoLuc could indeed generate enough bioluminescence to trigger miniSOG $\mathrm{PM}_{\mathrm{P}}$ photodynamic CCK1R activation, as shown below (Figure 2).

In expression plasmid pminiSOG $G_{P M}$-IRES-NanoLuc, an IRES sequence was inserted in between miniSOG $_{P M}$ and NanoLuc (Figure 2a). Confocal imaging confirmed the expression of miniSOG ${ }_{P M}$ (Figure 2b). NanoLuc expression (i.e., bioluminescence light emission) was readily detected after addition of substrate coelenterazine $5 \mu \mathrm{M}$ in miniSOG PM -IRES-NanoLuc-AR4-2J cells, but no bioluminescence was detected at all in a buffered solution of coelenterazine $5 \mu \mathrm{M}$ alone, in miniSOG ${ }_{P M}$-IRES-NanoLuc-AR4-2J cells without coelenterazine addition, or with coelenterazine $5 \mu \mathrm{M}$ addition to miniSOG $\mathrm{PM}^{-}$-AR4-2J cells not expressing NanoLuc (Figure 2c).

Tandem doses of CCK 10 pM induced reproducible calcium oscillations in NanoLuc-AR4-2J cells, and CCK-induced calcium oscillations disappeared immediately after wash-out of CCK; the addition of NanoLuc substrate coelenterazine $5 \mu \mathrm{M}$ in between the two CCK doses had no effect (Figure 2d). Sequential CCK $10 \mathrm{pM}$ also induced robust calcium oscillations in miniSOG $\mathrm{PM}^{-A R 4-2 J}$ cells; the addition of coelenterazine $5 \mu \mathrm{M}$ in between had no effect on baseline calcium concentration either (Figure 2e).

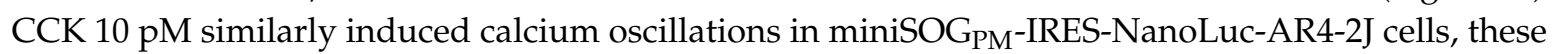
CCK-induced calcium oscillation peaks disappeared completely as expected after wash-out of CCK; subsequent addition of coelenterazine $5 \mu \mathrm{M}$ to these same cells induced calcium oscillations that were persistently present long after wash-out of the added coelenterazine (Figure 2f). These data indicate that, in the absence of an external light source, the addition of NanoLuc substrate coelenterazine $5 \mu \mathrm{M}$ after simultaneous expression of NanoLuc and miniSOG ${ }_{P M}$ in the CCK1R-expressing AR4-2J cells by

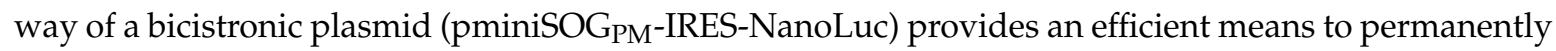
activate the endogenously expressed CCK1R. 
$\boldsymbol{a}$

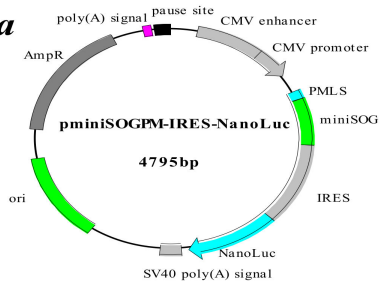

b

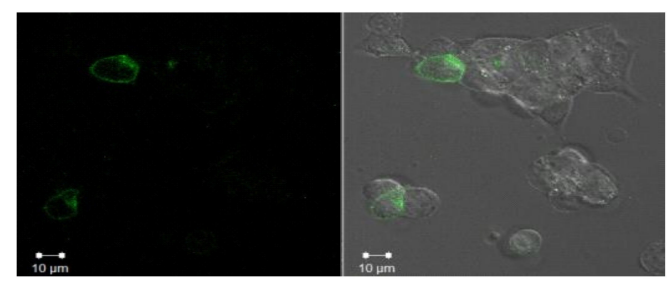

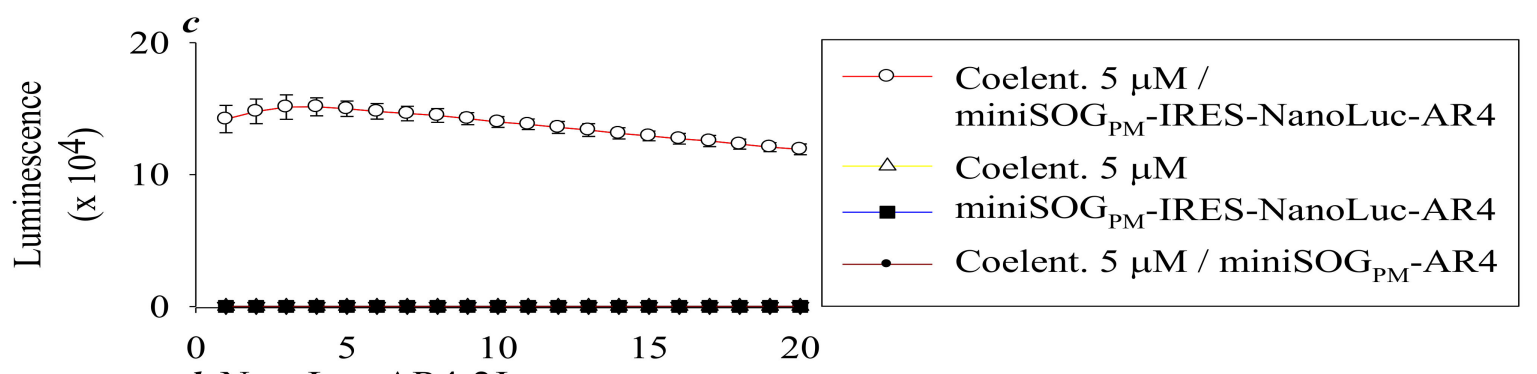

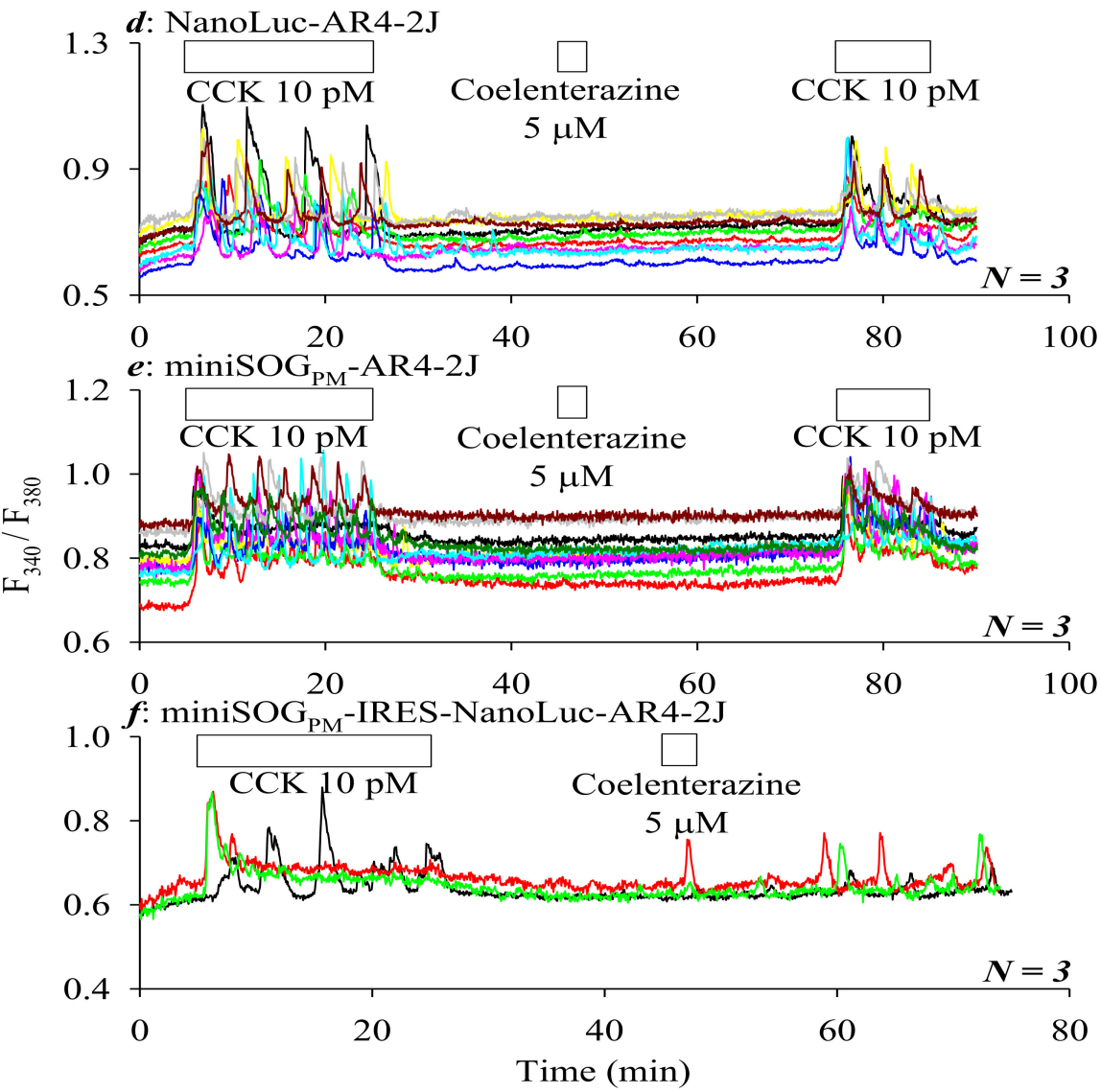

Figure 2. MiniSOGPM photodynamic CCK1R activation in AR4-2J cells driven by NanoLuc bioluminescence light. (a) Plasmid pminiSOG ${ }_{P M}-I R E S-N a n o L u c$. PMLS: plasma membrane-localizing

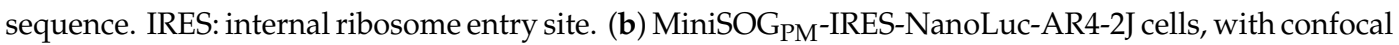
images $\left(\lambda_{\mathrm{ex}} 488 \mathrm{~nm}\right.$ ) taken $24 \mathrm{~h}$ after transfection with pminiSOG ${ }_{P M}$-IRES-NanoLuc. (c) Bioluminescene light emitted after addition of NanoLuc substrate coelenterazine $5 \mu \mathrm{M}$, no bioluminescence light was detected under control conditions (as indicated) (mean $\pm \mathrm{SEM}, n=3$ ). (d-f) Fura-2-loaded

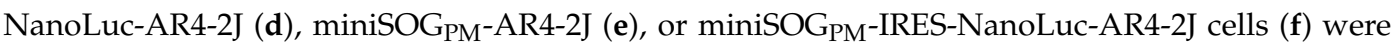
perifused, and CCK $(10 \mathrm{pM})$ and coelenterazine $5 \mu \mathrm{M}(3 \mathrm{~min})$ were applied as indicated by the horizontal bars. Colored calcium tracings are each from individual cells measured simultaneously. These tracings are from 1 out of $N$ identical experiments $(\mathbf{d}-\mathbf{f}, n=3)$. 


\section{Discussion}

In the present work, the GEPP miniSOG was expressed at the plasma membrane in rat pancreatic acinar tumor cell AR4-2J, and light irradiation with a blue LED $(450 \mathrm{~nm})$ light source of the miniSOG ${ }_{\mathrm{PM}}$-AR4-2J cells triggered long-lasting cytosolic calcium oscillations that were blocked completely by CCK1R antagonist devazepide. Therefore, miniSOG photodynamic action of CCK1R could be powered not only with a halogen cold light source as reported by us previously [33], but also with a wavelength-defined LED light source at $450 \mathrm{~nm}$. Further, both miniSOGPM and NanoLuc were expressed simultaneously in AR4-2J cells by transduction with a bicistronic plasmid pminiSOG $_{P M}$-IRES-NanoLuc with the insertion between the gene sequences of miniSOG and NanoLuc of an internal ribosome entry site (IRES) sequence. The resultant miniSOG ${ }_{P M}$-IRES-NanoLuc-AR4-2J cells were found to emit strong NanoLuc bioluminescence light upon addition of NanoLuc substrate coelenterazine. The addition of coelenterazine to perifused miniSOG ${ }_{\mathrm{PM}}$-IRES-NanoLuc-AR4-2J cells was found to trigger long-lasting cytosolic calcium oscillations that persisted long after wash-out of the added coelenterazine. These data together suggest that miniSOG photodynamic activation of CCK1R could be powered not only with a blue LED light source, but also with NanoLuc bioluminescence. The bicistronic plasmid pminiSOG ${ }_{P M}$ IRES-NanoLuc method could potentially be used to study photodynamic CCK1R physiology or pharmacology of CCK1R-expressing cells in vivo either in the CNS or in peripheral tissues.

CCK1R is unique among the dozen or so calcium-mobilizing A class GPCRs we have examined (not shown) in that it is activated permanently by ${ }^{1} \mathrm{O}_{2}$ generated in a type II photodynamic action with SALPC, GdPLMB, KillerRed, or miniSOG as the photosensitizer, all powered by a halogen cold light source, with the full-spectrum white light directly for KillerRed and miniSOG, or with red light after filtering out shorter wavelengths by a long-pass filter ( $>580 \mathrm{~nm}$ ) for SALPC and GdPLMB [29-31,33]. In the present work, we have extended our previous findings by powering miniSOG $_{P M}$ photodynamic activation of CCK1R with a blue LED $(450 \mathrm{~nm})$ as an external light source, and with NanoLuc bioluminescence light after addition of NanoLuc substrate coelenterazine to miniSOG ${ }_{P M}-N a n o L u c-A R 4-2 J$ cells as an internal (within the AR4-2J cells) light source.

MiniSOG was targeted to plasma membrane in AR4-2J cells, to emit green fluorescence $(500 \mathrm{~nm})$ (Figure 1a,b). Light irradiation with blue LED $\left(450 \mathrm{~nm}, 85 \mathrm{~mW} \cdot \mathrm{cm}^{-2}, 1.5 \mathrm{~min}\right)$ had no effect in parental AR4-2J cells (Figure 1c), but triggered long-lasting calcium oscillations in miniSOG ${ }_{P_{M}}$-AR4-2J cells (Figure 1e), which were similar to calcium oscillations induced in parallel experiments in untransfected AR4-2J cells by photodynamic action with chemical photosensitizer SALPC $(1 \mu \mathrm{M})$, triggered by red light ( $>580 \mathrm{~nm}, 36.7 \mathrm{~mW} \cdot \mathrm{cm}^{-2}, 1.5 \mathrm{~min}$ ) from the halogen cold light source (Figure 1d). CCK1 receptor antagonist devazepide $2 \mathrm{nM}$ completely blocked blue LED light irradiation-induced calcium oscillations in miniSOG ${ }_{P M}$-AR4-2J cells, unambiguously confirming blue LED-driven miniSOG ${ }_{P M}$ photodynamic activation of CCK1R (Figure 1f).

NanoLuc (19 kDa), a miniaturized luciferase originally from deep sea shrimp Oplophorus gracilirostris, could use either coelenterazine or synthetic furimazine as substrate, to emit blue light peaking at 478 and $460 \mathrm{~nm}$, respectively [41,42]. The emitted blue bioluminescence light is readily absorbed by miniSOG $\left(\lambda_{\mathrm{ex}} 448 \mathrm{~nm}\right.$ ) [34-36] (see text below and Table A1 for further details). NanoLuc has actually been used before quite widely to study protein-protein interactions [43], to regulate gene expression and cellular signaling [44,45], for molecular imaging [46,47], or for photodynamic killing of cancer cells [48,49]. The fusion construct NanoLuc-miniSOG with furimazine as substrate has been used to induce cancer cell killing in vitro [48-51]. It may be noted that, in these latter works, NanoLuc and miniSOG were expressed as single fused proteins NanoLuc-miniSOGand NanoLuc-[GGGGS]-miniSOG [48,50]. Emission spectral studies confirmed NanoLuc-miniSOG bioluminescence resonance energy transfer (BRET) in expressing human breast adenocarcinoma cells SK-BR-3, because NanoLuc bioluminescence excitation of miniSOG fluorescence was readily detected. Further, NanoLuc bioluminescence was able to power miniSOG photodynamic SK-BR-3 cell killing $[48,50]$. In our own work with NanoLuc-IRES-miniSOG-AR4-2J cells, it is likely that NanoLuc 
and miniSOG were expressed as separate proteins. NanoLuc distribution or accumulation under the miniSOG-expressing PM would provide the platform for PM-delimited NanoLuc to miniSOG BRET and therefore miniSOGPM photodynamic CCK1R activation (Figure 3). This NanoLuc to miniSOG $_{P M}$ BRET process probably needs to be further established, preferably in single cell microscopic spectral studies.

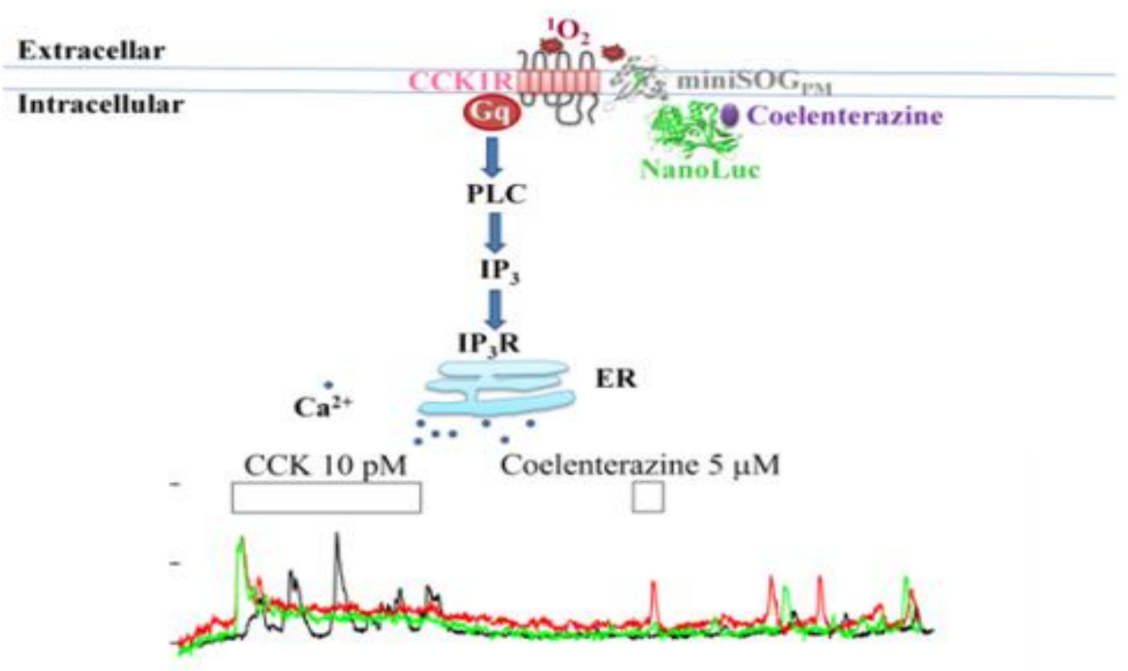

Figure 3. NanoLuc bioluminescence-driven miniSOGPM photodynamic CCK1R activation. MiniSOG was expressed at the plasma membrane (PM), in a bicistronic miniSOG PM $^{-I R E S-N a n o L u c}$ construct, resulting in the expression of separate miniSOG ${ }_{P M}$ and NanoLuc proteins. The addition

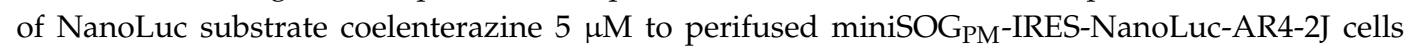
triggers the miniSOG $G_{P M}$ generation of singlet oxygen $\left({ }^{1} \mathrm{O}_{2}\right)$, likely via a radiationless NanoLuc to miniSOGPM BRET process; ${ }^{1} \mathrm{O}_{2}$ then oxidatively activates the endogenously expressed CCK1R in rat pancreatic acinar tumor cell AR4-2J. Gq, G protein q subtype; PLC, phospholipase C; IP3R, IP3 receptors; ER, endoplasmic reticulum.

In the present work, simultaneous expression of miniSOG ${ }_{P M}$ and NanoLuc in the rat pancreatic acinar tumor cell AR4-2J was done with a bicistronic plasmid pminiSOG ${ }_{P M}-I R E S-N a n o L u c$. Addition of NanoLuc substrate coelenterazine $5 \mu \mathrm{M}$ to miniSOG PM -IRES-NanoLuc-AR4-2J cells (with separate expression of miniSOG ${ }_{\mathrm{PM}}$ and NanoLuc) led to strong bioluminescence light emission, which was not found in control experiments (coelenterazine alone; miniSOG ${ }_{\mathrm{PM}}$-IRES-NanoLuc-AR4-2J cells

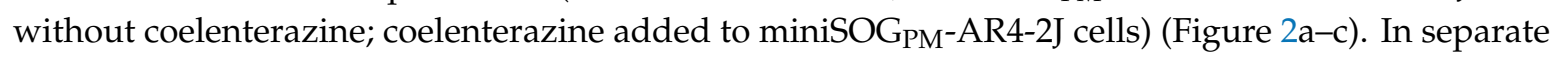

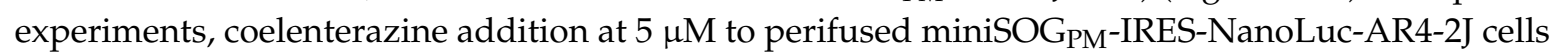
was found to trigger persistent and long-lasting calcium oscillations-a hallmark of miniSOG ${ }_{P M}$ photodynamic permanent activation of CCK1R (Figure 2f), but in NanoLuc-AR4-2J cells or miniSOG ${ }_{P M}$-AR4-2J cells, coelenterazine addition had no effect (Figure 2d,e). These data indicate

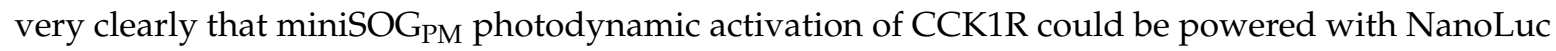

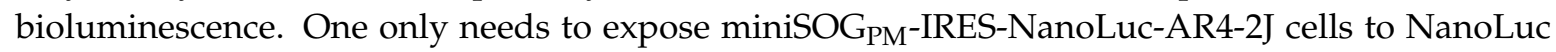
substrate coelenterazine at a relatively low concentration in the low $\mu \mathrm{M}$ range (Figure 3).

It may be noted that, for NanoLuc bioluminescence-driven miniSOGPM photodynamic activation of CCK1R, NanoLuc substrates other than coelenterazine might also be used. In AR4-2J cells, however, the synthetic substrate furimazine may not be used, because low micromolar furimazine concentrations (such as at $5 \mu \mathrm{M}$ ) alone induced calcium increases in the absence of NanoLuc expression (data not shown).

It may be further noted that, although NanoLuc with coelenterazine emits at $478 \mathrm{~nm}$ maximally $(100 \%)$, at $448 \mathrm{~nm}$, the emission is still at $68 \%$ of the maximum [42]. MiniSOG is excited maximally at $448 \mathrm{~nm}(100 \%)$, but at $478 \mathrm{~nm}$, the excitation spectrum is still at $77 \%$ of the maximum [35]. Therefore, there should be no problem for NanoLuc bioluminescence to excite miniSOG, for miniSOG 
photodynamic activation of CCK1R, which we observed as persistent cytosolic calcium oscillations (Figure 2f). Our work indicated that a match of NanoLuc maximal emission to $77 \%$ miniSOG excitation plus a match of $68 \%$ NanoLuc emission to miniSOG maximal excitation was sufficient (Table A1); an exact maximum-to-maximum or peak-to-peak match of the two is not necessary.

In conclusion, the recently emerged GEPP miniSOG could be used to photodynamically activate CCK1R permanently, not only with an external light source blue LED $(450 \mathrm{~nm})$ at a power density of $85 \mathrm{mWatt} . \mathrm{cm}^{-2}$ (1.5 min), but also with an internal light source in the form of simultaneously expressed NanoLuc upon the addition of NanoLuc substrate coelenterazine at $5 \mu \mathrm{M}(3 \mathrm{~min}$ ) (Figure 3) (see also Table A2, for a comparison of the parameters of these light sources). NanoLuc bioluminescence-powered permanent photodynamic CCK1R activation could be readily used for future in vivo applications without the need for cumbersome and complex optical delivery devices connected to an external light source, but with rather similar results in terms of permanent photodynamic CCK1R activation as with an external LED light source. This might lay the foundation for extensive studies of GEPP photodynamic CCK1R or other GPCR physiology or pharmacology.

\section{Materials and Methods}

\subsection{Materials}

Sulphated cholecystokinin octapeptide (CCK) and CCK1R antagonist devazepide were from Tocris Cookson (Bristol, UK). Dulbecco's Modified Eagle's Medium (DMEM) / F12 medium was bought from InVitrogen (Shanghai, China). Goods buffer 4-(2-hydroxyethyl)-1-piperazine-ethane-sulfonic acid (HEPES) was from Calbiochem (Darmstadt, Germany). Fura-2 AM was from AAT Bioquest (Sunnyvale, CA, USA). JetPRIME transfection reagent was from PolyPlus-transfection (New York, USA). Sulphonated aluminium phthalocyanine (SALPC) was from Frontier Scientific Inc. (AlPcS-834, Logan, UT, USA). Cell-Tak and Agar (Bacto ${ }^{\mathrm{TM}}$ ) were from BD Biosciences (Bedford, MA, USA). Fetal bovine serum (FBS) was from Thermo Scientific (Shanghai, China). $p$ KillerRed $_{P M}$ vector was bought from Evrogen (Moscow, Russia). pNL1.1.CMV (NanoLuc / CMV) vector containing the NanoLuc luciferase gene under the control of the cytomegalovirus (CMV) promoter and coelenterazine were from Promega (Madison, WI, USA). PEF1A-IRES-Neo vector was from Addgene (Watertown, MA, USA). Ampicillin and Kanamycin were from CWBio (Beijing, China). Endotoxin-free plasmid extraction kit and DH5à competent cells were from TianGen Biochemicals (Beijing, China).

\subsection{AR4-2J and E. coli Cell Culture}

AR4-2J was bought from ATCC (Rockville, MD, USA) and cultured in DMEM/F12 supplemented with $20 \%$ fetal bovine serum in a $\mathrm{CO}_{2}$ incubator under humidified atmosphere $\left(5 \% \mathrm{CO}_{2} / 95 \%\right.$ air $)$ at $37^{\circ} \mathrm{C}$, as reported before $[1,2,28,33,52]$.

Solid E. coli medium LB/Kana and LB/Amp were sterilized and culture plates made. Liquid E. coli medium LB/Kana and LB/Amp had the same composition, but without agar.

\subsection{Vector Constructs}

A mammalian codon-optimized miniSOG gene (GenBank accession number JX999997) was synthesized de novo from nucleotides at Genscript (Nanjing, China) with the following full nucleotide sequence: ATGGAAAAGAGCTTTGTGATTACCGATCCGCGCCTGCCAGACAACCCG ATCATTTTCGCGAGCGATGGCTTTCTGGAGTTAACCGAATATTCTCGTGAGGAAATTCTGGGTCG CAATGGCCGTTTCTTGCAGGGTCCGGAAACGGATCAAGCCACCGTGCAGAAAATCCGCGATGC GATTCGTGACCAACGCGAAATCACCGTTCAGCTGATTAACTATACGAAAAGCGGCAAGAAATT TTGGAACTTACTGCATCTGCAACCGATGCGCGATCAGAAAGGCGAATTGCAATATTTCATTGGT GTGCAGCTGGATGGCTAG. This synthesized full miniSOG gene sequence was inserted into plasmid pKillerRed $_{P M}$ (Evrogen, Moscow, Russia), to replace the KillerRed sequence. Competent E. coli were infected with the recombinant plasmid, cultured on solid LB/kana. Bacteria colonies were picked and 
further cultured in liquid LB/kana with shaking overnight. Proliferated plasmid was extracted for sequence verification. The obtained plasmid was named pminiSOG $G_{P M}$ owing to the presence of a plasma membrane (PM) localization sequence in the original Evrogen plasmid. The transfected AR4-2J cells were named miniSOGPM-AR4-2J cells.

For construction of pminiSOG ${ }_{P M}$-IRES-NanoLuc, the coding sequence of miniSOG $G_{P M}$ was cloned into plasmid pNL1.1.CMV (NanoLuc/CMV) (Promega) containing the NanoLuc luciferase gene under the control of the CMV promoter (Genscript, Nanjing, China). The miniSOG $P M$ sequence was amplified from pminiSOG $_{P M}$ plasmid. The IRES sequence amplified from PEF1A-IRES-Neo vector was inserted between the two functional domains (NanoLuc and miniSOG ${ }_{P M}$ ). The plasmid construct was transformed into DH5a, harvested, and sequenced for verification. This plasmid was named pminiSOG ${ }_{P M}$-IRES-NanoLuc, and transduced AR4-2J cells were named miniSOG ${ }_{P M}$-IRES-NanoLuc-AR4-2J, where both miniSOG ${ }_{P M}$ and NanoLuc would express as separate proteins.

\subsection{Transduction of AR4-2J Cells}

AR4-2J cells were cultured in six-well plates or six-well plates with a round glass cover-slip in each well, and cells were allowed to grow to $50-70 \%$ confluence. Mixed plasmid ( $2 \mu \mathrm{g} / \mathrm{well})$ and jetPRIME transfection reagent $(4 \mu \mathrm{L} /$ well $)$ in jetPRIME buffer $(200 \mu \mathrm{L})$ were added before the cells were cultured for a further $24 \mathrm{~h}$. Transfection of AR4-2J cells was verified by NanoLuc bioluminescence (Tecan Infinite F200 Pro multimode reader) or miniSOG fluorescence confocal imaging (Zeiss LSM510 META, objective lens oil $\times 60$ ).

\section{5. miniSOG Fluorescence Imaging}

Parental AR4-2J cells were planted on glass cover-slips and cultured overnight before transfection and further cultivation. At $24 \mathrm{~h}$ after transfection, cells were imaged in a confocal microscope (Zeiss LSM 510 META), under oil objective $63 \times / 1.40$, with $\lambda_{\text {ex }}$ for miniSOG at $488 \mathrm{~nm}$.

\subsection{Photodynamic Action}

To trigger photodynamic action, SALPC-bound cells were irradiated with red light (>580 nm, $36.7 \mathrm{~mW} \cdot \mathrm{cm}^{-2}, 1.5 \mathrm{~min}$ ) from a halogen cold light source (MegaLight 100, Hoya-Schott, Japan) equipped with condenser HLL201 and filter R60 (>80 nm).

MiniSOG PM $^{-A R 4-2 J ~ c e l l s ~ w e r e ~ i r r a d i a t e d ~ w i t h ~ a ~ b l u e ~ L E D ~}\left(450 \mathrm{~nm}, 85 \mathrm{~mW} \cdot \mathrm{cm}^{-2}, 1.5 \mathrm{~min}\right)$ (LAMPLIC, Shenzhen, China). Light irradiance at the level of attached cells in the Sykes-Moore perfusion chamber was measured with a power meter (IL1700, International Light Inc., Newburyport, MA, USA).

For NanoLuc bioluminescene to power miniSOGPM photodynamic action, NanoLuc bioluminescence was first characterized and recorded in a Tecan Infinite F200 Pro multimode reader after addition of NanoLuc substrate coelenterazine. Measurements were carried out on living cells in a balanced buffer in 96-well plates with white walls (in triplicates). NanoLuc substrate coelenterazine (Promega) was added at a final concentration of $5 \mu \mathrm{M}$. Luminescence was recorded once every min. Coelenterazine $5 \mu \mathrm{M}$ was added to perifused miniSOG ${ }_{\mathrm{PM}}$-IRES-NanoLuc-AR4-2J cells ( $3 \mathrm{~min}$ ) to trigger NanoLuc bioluminescence-powered miniSOG ${ }_{P M}$ photodynamic CCK1R activation.

\subsection{Calcium Measurements}

Transfected AR4-2J cells grown on glass cover-slips in six-well plates were loaded with Fura-2 AM (final concentration $10 \mu \mathrm{M}, 1 \mathrm{~h}$ ) directly after assembly in Sykes-Moore perfusion chambers. Cytosolic calcium was measured in an inverted fluorescent microscope (Nikon TE-2000U) coupled to a PTI (Photon Technology International Inc., now HORIBA, Edison, NJ, USA) calcium measurement system with alternating excitations of the loaded Fura-2 at $340 \mathrm{~nm} / 380 \mathrm{~nm}$ (DeltaRam X), and emitted Fura-2 fluorescence (dichroic mirror 400DCLP, emitter $510 \pm 40 \mathrm{~nm}$ ) was detected with a charge-coupled device (CCD) camera (NEO-5.5-CL-3, Andor/Oxford Instruments, UK). Calcium concentration was expressed 
as $F_{340} / F_{380}$ ratios and plotted against time with SigmaPlot, as reported before $[1,2,28,31,33,52,53]$. In the figures shown in Results, the original colored calcium tracings were each from individual cells and only representative tracings from 1 out of $N$ (as indicated, $N \geq 3$ ) identical experiments were presented.

\subsection{Data Presentation and Statistical Analysis}

All data were presented as mean \pm SEM. Student's T-test was used for statistical analysis against controls and $p<0.05$ was taken as statistically significant, as indicated $(*)$. For calculation and comparison of induced calcium responses, calcium peak area above the baseline was integrated (usually per 10 min unless stated otherwise). All calcium tracings (each line tracing corresponds to an individual cell) and other data graphs were plotted with SigmaPlot.

Author Contributions: Conceptualization, Z.J.C.; Methodology, Z.J.C.; Formal Analysis, Y.L. and Z.J.C.; Investigation, Y.L.; Resources, Z.J.C.; Data Curation, Y.L.; Writing-Original Draft Preparation, Y.L.; Writing-Review \& Editing, Z.J.C.; Visualization, Y.L. and Z.J.C.; Supervision, Z.J.C.; Funding Acquisition, Z.J.C. All authors have read and agree to the published version of the manuscript.

Funding: This research was funded by The Natural Science Foundation of China, grant numbers 31670856, 31971170 , and by a previous grant from The Ministry of Science and Technology of China (973 program), grant number 2011CB809101. The APC was funded by The Natural Science Foundation of China.

Conflicts of Interest: The authors declare no conflict of interest. The funders had no role in the design of the study; in the collection, analyses, or interpretation of data; in the writing of the manuscript; or in the decision to publish the results.

\section{Abbreviations}

$\begin{array}{ll}\text { CCK1R } & \text { Cholecystokinin 1 receptors } \\ \text { CNS } & \text { Central nervous system } \\ \text { GdPLMB } & \text { Gadolinium porphyrin-like macrocycle B } \\ \text { GEPP } & \text { Genetically-encoded protein photosensitizer } \\ \text { GPCR } & \text { G protein-coupled receptor } \\ \text { IRES } & \text { Internal ribosome entry site } \\ \text { miniSOG } & \text { mini singlet oxygen generator } \\ { }_{\text {LED }} & \text { Light-emitting diode } \\ { }^{1} \mathrm{O}_{2} & \text { Singlet oxygen } \\ \text { PM } & \text { Plasma membrane } \\ \text { SALPC } & \text { Sulphonated aluminium phthlaocyanine }\end{array}$

\section{Appendix A Aix A}

Table A1. Comparison of NanoLuc emission and mini singlet oxygen generator (miniSOG) excitation parameters.

\begin{tabular}{cccccc}
\hline & NanoLuc [42] & \multicolumn{3}{c}{ miniSOG [35] } \\
\hline Peak & $478 \mathrm{~nm}$ & $100 \%$ & $478 \mathrm{~nm}$ & $77 \%$ \\
\hline & $448 \mathrm{~nm}$ & $68 \%$ & Peak & $448 \mathrm{~nm}$ & $100 \%$ \\
\hline
\end{tabular}

These parameters were derived from figures in $[35,42]$. 
Table A2. Light sources to power miniSOGPM photodynamic cholecystokinin 1 receptor (CCK1R) activation. LED, light-emitting diode.

\begin{tabular}{cccc}
\hline Light Sources & Power Density & Duration (min) & References \\
\hline Halogen cold light $($ white $)$ & $87 \mathrm{~mW} \cdot \mathrm{cm}^{-2}$ & 5 & {$[33]$} \\
\hline Blue LED $(450 \mathrm{~nm})$ & $85 \mathrm{~mW} \cdot \mathrm{cm}^{-2}$ & 1.5 & This work \\
\hline NanoLuc + coelenterazine & $5 \mu \mathrm{M}$ (coelenterazine) & 3 & This work \\
\hline
\end{tabular}

\section{References}

1. Guo, H.Y.; Cui, Z.J. Extracellular histones activate plasma membrane Toll-like receptor 9 to trigger calcium oscillations in rat pancreatic acinar tumor cell AR4-2J. Cells 2019, 8, 3. [CrossRef] [PubMed]

2. Liu, J.S.; Cui, Z.J. Pancreatic stellate cells serve as a brake mechanism on pancreatic acinar cell calcium signaling modulated by methionine sulfoxide reductase expression. Cells 2019, 8, 109. [CrossRef] [PubMed]

3. Mercer, L.D.; Beart, P.M. Histochemistry in rat brain and spinal cord with an antibody directed at the cholecystokinin A receptor. Neurosci. Lett. 1997, 225, 97-100. [CrossRef]

4. Mercer, L.D.; Beart, P.M. Immunolocalization of CCK1R in rat brain using a new anti-peptide antibody. Neurosci. Lett. 2004, 359, 109-113. [CrossRef]

5. Honda, T.; Wada, E.; Battey, J.F.; Wank, S.A. Differential gene expression of CCKA and CCKB receptors in the rat brain. Mol. Cell Neurosci. 1993, 4, 143-154. [CrossRef]

6. Nishimura, S.; Bilgüvar, K.; Ishigame, K.; Sestan, N.; Günel, M.; Louvi, A. Functional synergy between cholecystokinin receptors CCKAR and CCKBR in mammalian brain development. PLoS ONE 2015, 10, e0124295. [CrossRef]

7. Broberger, C.; Holmberg, K.; Shi, T.J.; Dockray, G.; Hökfelt, T. Expression and regulation of cholecystokinin and cholecystokinin receptors in rat nodose and dorsal root ganglia. Brain Res. 2001, 903, 128-140. [CrossRef]

8. Glatzle, J.; Wang, Y.; Adelson, D.W.; Kalogeris, T.J.; Zittel, T.T.; Tso, P.; Wei, J.Y.; Raybould, H.E. Chylomicron components activate duodenal vagal afferents via a cholecystokinin A receptor-mediated pathway to inhibit gastric motor function in the rat. J. Physiol. 2003, 550, 657-664. [CrossRef]

9. Li, Y.; Wu, X.; Zhou, S.; Owyang, C. Low-affinity CCK-A receptors are coexpressed with leptin receptors in rat nodose ganglia: Implications for leptin as a regulator of short-term satiety. Am. J. Physiol. Gastrointest. Liver Physiol. 2011, 300, G217-G227. [CrossRef]

10. Mussa, B.M.; Sartor, D.M.; Verberne, A.J. Activation of cholecystokinin (CCK 1) and serotonin (5-HT 3) receptors increases the discharge of pancreatic vagal afferents. Eur. J. Pharmacol. 2008, 601, 198-206. [CrossRef]

11. Patterson, L.M.; Zheng, H.; Ward, S.M.; Berthoud, H.R. Immunohistochemical identification of cholecystokinin A receptors on interstitial cells of Cajal, smooth muscle, and enteric neurons in rat pylorus. Cell Tissue Res. 2001, 305, 11-23. [CrossRef] [PubMed]

12. Patterson, L.M.; Zheng, H.; Berthoud, H.R. Vagal afferents innervating the gastrointestinal tract and CCKA-receptor immunoreactivity. Anat. Rec. 2002, 266, 10-20. [CrossRef] [PubMed]

13. Tsujino, N.; Yamanaka, A.; Ichiki, K.; Muraki, Y.; Kilduff, T.S.; Yagami, K.; Takahashi, S.; Goto, K.; Sakurai, T. Cholecystokinin activates orexin/hypocretin neurons through the cholecystokinin A receptor. J. Neurosci. 2005, 25, 7459-7469. [CrossRef] [PubMed]

14. Ruiz-Gayo, M.; Garrido, M.M.; Fuentes, J.A. Inhibition of the hypothalamic-pituitary-adrenal axis in food-deprived rats by a CCK-A receptor antagonist. Br. J. Pharmacol. 2000, 129, 839-842. [CrossRef] [PubMed]

15. Hashimoto, H.; Onaka, T.; Kawasaki, M.; Chen, L.; Mera, T.; Soya, A.; Saito, T.; Fujihara, H.; Sei, H.; Morita, Y.; et al. Effects of cholecystokinin (CCK)-8 on hypothalamicoxytocin-secreting neurons in rats lacking CCK-A receptor. Auton. Neurosci. 2005, 121, 16-25. [CrossRef] [PubMed]

16. Yamamoto, Y.; Akiyoshi, J.; Kiyota, A.; Katsuragi, S.; Tsutsumi, T.; Isogawa, K.; Nagayama, H. Increased anxiety behavior in OLETF rats without cholecystokinin-A receptor. Brain Res. Bull. 2000, 53, 789-792. [CrossRef] 
17. Wen, D.; Sun, D.; Zang, G.; Hao, L.; Liu, X.; Yu, F.; Ma, C.; Cong, B. Cholecystokinin octapeptide induces endogenous opioid-dependent anxiolytic effects in morphine-withdrawal rats. Neuroscience 2014, 277, $14-25$. [CrossRef]

18. Zhu, G.; Yan, J.; Smith, W.W.; Moran, T.H.; Bi, S. Roles of dorsomedial hypothalamic cholecystokinin signaling in the controls of meal patterns and glucose homeostasis. Physiol. Behav. 2012, 105, 234-241. [CrossRef]

19. Sui, Y.; Vermeulen, R.; Hokfelt, T.; Horne, M.K.; Stanic, D. Female mice lacking cholecystokinin 1 receptors have compromised neurogenesis, and fewer dopaminergic cells in the olfactory bulb. Front. Cell Neurosci. 2013, 7, 13. [CrossRef]

20. Matsushita, H.; Akiyoshi, J.; Kai, K.; Ishii, N.; Kodama, K.; Tsutsumi, T.; Isogawa, K.; Nagayama, H. Spatial memory impairment in OLETF rats without cholecystokinin-A receptor. Neuropeptides 2003, 37, 271-276. [CrossRef]

21. Suzuki, S.; Takiguchi, S.; Sato, N.; Kanai, S.; Kawanami, T.; Yoshida, Y.; Miyasaka, K.; Takata, Y.; Funakoshi, A.; Noda, T. Importance of CCK-A receptor for gallbladder contraction and pancreatic secretion: A study in CCK-A receptor knockout mice. Jpn. J. Physiol. 2001, 51, 585-590. [CrossRef] [PubMed]

22. Takiguchi, S.; Suzuki, S.; Sato, Y.; Kanai, S.; Miyasaka, K.; Jimi, A.; Shinozaki, H.; Takata, Y.; Funakoshi, A.; et al. Role of CCK-A receptor for pancreatic function in mice: A study in CCK-A receptor knockout mice. Pancreas 2002, 24, 276-283. [CrossRef] [PubMed]

23. Malendowicz, L.K.; Nowak, M.; Gottardo, L.; Tortorella, C.; Majchrzak, M.; Nussdorfer, G.G. Cholecystokinin stimulates aldosterone secretion from dispersed rat zona glomerulosa cells, acting through cholecystokinin receptors 1 and 2 coupled with the adenylate cyclase-dependent cascade. Endocrinology 2001, 142, 4251-4255. [CrossRef] [PubMed]

24. Whited, K.L.; Thao, D.; Lloyd, K.C.; Kopin, A.S.; Raybould, H.E. Targeted disruption of the murine CCK1 receptor gene reduces intestinal lipid-induced feedback inhibition of gastric function. Am. J. Physiol. Gastrointest. Liver Physiol. 2006, 291, G156-G162. [CrossRef]

25. Boeckxstaens, G.E.; Hirsch, D.P.; Fakhry, N.; Holloway, R.H.; D'Amato, M.; Tytgat, G.N. Involvement of cholecystokinin A receptors in transient lower esophageal sphincter relaxations triggered by gastric distension. Am. J. Gastroenterol. 1998, 93, 1823-1828. [CrossRef]

26. Varga, G.; Bálint, A.; Burghardt, B.; D’Amato, M. Involvement of endogenous CCK and CCK1 receptors in colonic motor function. Br. J. Pharmacol. 2004, 141, 1275-1284. [CrossRef]

27. Williams, J.A. Cholecystokinin (CCK) regulation of pancreatic acinar cells: Physiological actions and signal transduction mechanisms. Compr. Physiol. 2019, 9, 535-564. [CrossRef]

28. Liang, H.Y.; Song, Z.M.; Cui, Z.J. Lasting inhibition of receptor-mediated calcium oscillations in pancreatic acini by neutrophil respiratory burst-A novel mechanism for secretory blockade in acute pancreatitis? Biochem. Biophys. Res. Commun. 2013, 437, 361-367. [CrossRef]

29. Cui, Z.J.; Kanno, T. Photodynamic triggering of calcium oscillation in the isolated rat pancreatic acini. J. Physiol. 1997, 504, 47-55. [CrossRef]

30. Cui, Z.J.; Habara, Y.; Wang, D.Y.; Kanno, T. A novel aspect of photodynamic action: Induction of recurrent spikes in cytosolic calcium concentration. Photochem. Photobiol. 1997, 65, 382-386. [CrossRef]

31. An, Y.P.; Xiao, R.; Cui, H.; Cui, Z.J. Selective activation by photodynamic action of cholecystokinin receptor in the freshly isolated rat pancreatic acini. Br. J. Pharmacol. 2003, 139, 872-880. [CrossRef] [PubMed]

32. Jiang, H.N.; Li, Y.; Cui, Z.J. Photodynamic physiology—Photonanomanipulations in cellular physiology with protein photosensitisers. Front. Physiol. 2017, 8, 191. [CrossRef] [PubMed]

33. Jiang, H.N.; Li, Y.; Cui, Z.J. Cholecystokinin 1 receptor-a unique G protein-coupled receptor activated by singlet oxygen (GPCR-ABSO). Front. Physiol. 2018, 9, 497. [CrossRef] [PubMed]

34. Shu, X.; Lev-Ram, V.; Deerinck, T.J.; Qi, Y.; Ramko, E.B.; Davidson, M.W.; Jin, Y.; Ellisman, M.H.; Tsien, R.Y. A genetically encoded tag for correlated light and electron microscopy of intact cells, tissues, and organisms. PLoS Biol. 2011, e1001041. [CrossRef] [PubMed]

35. Pimenta, F.M.; Jensen, R.L.; Breitenbach, T.; Etzerodt, M.; Ogilby, P.R. Oxygen-dependent photochemistry and photophysics of "miniSOG," a protein-encased flavin. Photochem. Photobiol. 2013, 89, 1116-1126. [CrossRef] [PubMed]

36. Torra, J.; Lafaye, C.; Signor, L.; Aumonier, S.; Flors, C.; Shu, X.; Nonell, S.; Gotthard, G.; Royant, A. Tailing miniSOG: Structural bases of the complex photophysics of a flavin-binding singlet oxygen photosensitizing protein. Sci. Rep. 2019, 9, 2428. [CrossRef] 
37. Rodríguez-Pulido, A.; Cortajarena, A.L.; Torra, J.; Ruiz-González, R.; Nonell, S.; Flors, C. Assessing the potential of photosensitizing flavoproteins as tags for correlative microscopy. Chem. Commun. 2016, 52, 8405-8408. [CrossRef]

38. Endres, S.; Wingen, M.; Torra, J.; Ruiz-Gonzalez, R.; Polen, T.; Bosio, G.; Bitzenhofer, N.L.; Hilgers, F.; Gensch, T.; Nonell, S.; et al. An optogenetic toolbox of LOV-based photosensitizers for light-driven killing of bacteria. Sci. Rep. 2018, 8, 15021. [CrossRef]

39. Westberg, M.; Bregnhøj, M.; Etzerodt, M.; Ogilby, P.R. No photon wasted: An efficient and selective singlet oxygen photosensitizing protein. J. Phys. Chem. B 2017, 121, 9366-9371. [CrossRef]

40. Westberg, M.; Etzerodt, M.; Ogilby, P.R. Rational design of genetically encoded singlet oxygen photosensitizing proteins. Curr. Opin. Struct. Biol. 2019, 57, 56-62. [CrossRef]

41. Hall, M.P.; Unch, J.; Binkowski, B.F.; Valley, M.P.; Butler, B.L.; Wood, M.G.; Otto, P.; Zimmerman, K.; Vidugiris, G.; Machleidt, T.; et al. Engineered luciferase reporter from a deep sea shrimp utilizing a novel imidazopyrazinone substrate. ACS Chem. Biol. 2012, 7, 1848-1857. [CrossRef] [PubMed]

42. Shakhmin, A.; Hall, M.P.; Machleidt, T.; Walker, J.R.; Wood, K.V.; Kirkland, T.A. Coelenterazine analogues emit red-shifted bioluminescence with NanoLuc. Org. Biomol. Chem. 2017, 15, 8559-8567. [CrossRef] [PubMed]

43. Machleidt, T.; Woodroofe, C.C.; Schwinn, M.K.; Méndez, J.; Robers, M.B.; Zimmerman, K.; Otto, P.; Daniels, D.L.; Kirkland, T.A.; Wood, K.V. NanoBRET-A novel BRET platform for the analysis of protein-protein interactions. ACS Chem. Biol. 2015, 10, 1797-1804. [CrossRef] [PubMed]

44. Lackner, D.H.; Carré, A.; Guzzardo, P.M.; Banning, C.; Mangena, R.; Henley, T.; Oberndorfer, S.; Gapp, B.V.; Nijman, S.M.; Brummelkamp, T.R.; et al. A generic strategy for CRISPR-Cas9-mediated gene tagging. Nat. Commun. 2015, 6, 10237. [CrossRef]

45. Yasuzaki, Y.; Yamada, Y.; Ishikawa, T.; Harashima, H. Validation of mitochondrial gene delivery in liver and skeletal muscle via hydrodynamic injection using an artificial mitochondrial reporter DNA vector. Mol. Pharm. 2015, 12, 4311-4320. [CrossRef]

46. Stacer, A.C.; Nyati, S.; Moudgil, P.; Iyengar, R.; Luker, K.E.; Rehemtulla, A.; Luker, G.D. NanoLuc reporter for dual luciferase imaging in living animals. Mol. Imaging 2013, 12, 1-13. [CrossRef]

47. Germain-Genevois, C.; Garandeau, O.; Couillaud, F. Detection of brain tumors and systemic metastases using NanoLuc and Fluc for dual reporter imaging. Mol. Imaging Biol. 2016, 18, 62-69. [CrossRef]

48. Shramova, E.I.; Proshkina, G.M.; Chumakov, S.P.; Khodarovich, Y.M.; Deyev, S.M. Flavoprotein miniSOG cytotoxisity can be induced by bioluminescence resonance energy transfer. Acta Nat. 2016, 8, 118-123. [CrossRef]

49. Shramova, E.I.; Proshkina, G.M.; Deyev, S.M.; Petrov, R.V. Flavoprotein miniSOG BRET-induced cytotoxicity depends on its intracellular localization. Dokl. Biochem. Biophys. 2017, 474, 228-230. [CrossRef]

50. Proshkina, G.M.; Shramova, E.I.; Shilova, O.N.; Ryabova, A.V.; Deyev, S.M. Phototoxicity of flavoprotein miniSOG induced by bioluminescence resonance energy transfer in genetically encoded system NanoLuc-miniSOG is comparable with its LED-excited phototoxicity. J. Photochem. Photobiol. B Biol. 2018, 188, 107-115. [CrossRef]

51. Shramova, E.I.; Proshkina, G.M.; Deyev, S.M.; Petrov, R.V. Death mechanism of breast adenocarcinoma cells caused by BRET-induced cytotoxicity of miniSOG depends on the intracellular localization of the NanoLuc-miniSOG fusion protein. Dokl. Biochem. Biophys. 2018, 482, 288-291. [CrossRef] [PubMed]

52. Duan, Y.J.; Liang, H.Y.; Jin, W.J.; Cui, Z.J. Substance P conjugated to CdTe quantum dot triggers cytosolic calcium oscillations and induces QD internalization in the pancreatic carcinoma cell line AR4-2J. Anal. Bioanal. Chem. 2011, 400, 2995-3003. [CrossRef] [PubMed]

53. Fang, X.F.; Cui, Z.J. The anti-botulism triterpenoid toosendanin elicits calcium increase and exocytosis in rat sensory neurons. Cell Mol. Neurobiol. 2011, 31, 1151-1162. [CrossRef] [PubMed]

(C) 2020 by the authors. Licensee MDPI, Basel, Switzerland. This article is an open access article distributed under the terms and conditions of the Creative Commons Attribution (CC BY) license (http://creativecommons.org/licenses/by/4.0/). 